\title{
Transbronchial Lung Cryobiopsy in Diffuse Parenchymal Lung Disease: Comparison between Biopsy from 1 Segment and Biopsy from 2 Segments - Diagnostic Yield and Complications
}

\author{
Claudia Ravaglia ${ }^{a}$ Athol U. Wells ${ }^{g}$ Sara Tomassetti ${ }^{a}$ Alessandra Dubini $^{b}$

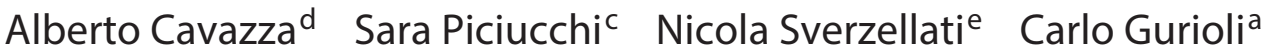 \\ ${\text { Christian Guriolia }{ }^{a} \text { Ulrich Costabel }}^{\text {h }}$ Paola Tantalocco ${ }^{a}$ Jay H. Ryu ${ }^{\mathrm{i}}$ \\ Marco Chilosi ${ }^{f}$ Venerino Polettia, j
}

\begin{abstract}
Departments of a Diseases of the Thorax, ${ }^{b}$ Pathology and ${ }^{\mathrm{C}}$ Radiology, G.B. Morgagni - L. Pierantoni Hospital, Forli, ${ }^{\mathrm{d}}$ Department of Pathology, S. Maria Nuova Hospital-I.R.C.C.S, Reggio Emilia, e'Division of Radiology, Department of Clinical Sciences, University of Parma, Parma, and f Department of Pathology, Verona University, Verona, Italy; gInterstitial Lung Disease Unit, Royal Brompton Hospital, London, UK; ${ }^{h}$ Ruhrlandklinik Essen, Universitätsklinikum Essen, Essen, Germany; ${ }^{i}$ Pulmonary and Critical Care Medicine, Mayo Clinic, Rochester, MN, USA; ${ }^{j}$ Department of Respiratory Medicine and Allergy, Aarhus University Hospital, Aarhus, Denmark
\end{abstract}

\section{Keywords}

Transbronchial lung cryobiopsy · Interstitial lung disease •

Samples · Lobes

\begin{abstract}
Background: Transbronchial lung cryobiopsy is an innovative method of obtaining samples from the parenchyma of patients with diffuse parenchymal lung diseases. However, the technique is not yet standardized, and uncertainty exists about the optimal protocol, including the number of samples, the biopsy size, and the choice of the biopsy site. Objectives: To compare the diagnostic yield and complications of cryobiopsy with different strategies adopted to sample lung tissue (number of samples, biopsy site, and sample size). Methods: We prospectively enrolled 46 patients with suspected diffuse parenchymal lung diseases for the diagnosis of which a biopsy was deemed useful. All patients under-
\end{abstract}

(c) 2017 S. Karger AG, Basel

\section{E-Mail karger@karger.com} www.karger.com/res went transbronchial lung cryobiopsy, and they were randomly assigned to group A (4 samples obtained from the same segment) or group B ( 2 samples obtained from one segment and 2 samples obtained from a different segment of the same lobe). Analysis of the samples was performed sequentially (from the first to the last sample), and pathologists reformulated their histopathologic diagnosis with the addition of each sample. Results: The mean diagnostic yield of the procedure combining the 2 groups and performing only the first sampling was $69 \%$. When a second biopsy was performed as well, the mean diagnostic yield improved, but this increase was significant only when the 2 samples were obtained from 2 different segments (96\%, group B). Conclusions: This study suggests that the strategy of performing 2 biopsies with a cryoprobe may be associated with an increased diagnostic yield in diffuse parenchymal lung diseases if these samples are obtained from 2 different segments within the same lobe.

(c) 2017 S. Karger AG, Basel 


\section{Introduction}

Transbronchial lung cryobiopsy (TLCB) is an innovative method of obtaining samples from the parenchyma of patients with diffuse parenchymal lung diseases (DPLD) [1-4]. Support for the use of TLCB with a DPLD diagnosis comes from a number of recent studies and editorials [5-15]. However, the technique is not yet standardized [5-16]. Two to 6 biopsies are usually taken [1,2, 17], but uncertainty exists about the optimal protocol, including the number of samples, the biopsy size, and the choice of the biopsy site (specifically, 1 segment only vs. 2 segments). Non-diagnostic findings could be more prevalent when smaller cryobiopsy specimens are taken [3], but the exact impact of the size of specimens on the diagnostic yield has yet to be quantified. It is widely accepted that surgical lung biopsies (SLB) should be taken from 2 different lobes to minimize sampling error, but it is not known whether restricting the acquisition of TLCB to a single lobar segment reduces the diagnostic yield.

In order to examine these questions, a prospective study was conducted. Our primary goal was to compare the diagnostic yield and the prevalence of complications between (a) performing all biopsies in the same lobar segment and (b) acquiring biopsies from 2 segments of the same lobe. We also examined the impact of increasing the total number of biopsies and increasing the biopsy size on the prevalence of non-diagnostic biopsies.

\section{Subjects and Methods}

Patients with DPLD with non-diagnostic clinical profiles, CT scan features, and laboratory tests (including autoimmune serology and precipitins) for whom a biopsy was deemed useful for a diagnosis were prospectively enrolled. Forty-six patients were enrolled between September 2014 and January 2015 at the Pulmonology Unit of G.B. Morgagni - L. Pierantoni Hospital, Forli, Italy. This prospective study was approved by the Area Vasta Romagna Ethics Committee (protocol No. 61144). Written informed consent for participation in the study was obtained from each patient.

The enrolled patients underwent TLCB and were randomly assigned to 2 different sampling strategies: strategy A (4 samples obtained from the same segment [A1, A2, A3, A4]) and strategy B (2 samples obtained from one segment [B1, B2] and 2 samples obtained from a different segment of the same lobe [B3, B4]).

The following clinical data were extracted: age, gender, past medical history, medications, smoking history, environmental exposure history, physical examination findings, laboratory results (including antinuclear autoantibodies, autoantibodies against extractable nuclear antigens, anti-neutrophil autoantibodies, and precipitins), and pulmonary function. The following were exclusion criteria: (a) coagulopathy (platelet count $<70,000 \times 10^{9} / \mathrm{L}$ or prothrombin time international normalized ratio $>1.5$ ); (b) FVC
$<50 \%$ of the predicted value, DLCO $<30 \%$ of the predicted value, and $\mathrm{FEV}_{1}<0.8 \mathrm{~L}$; (c) age $<18$ years; (d) diffuse bullous disease; (e) haemodynamic instability; (f) echocardiographic pulmonary arterial systolic pressure $>50 \mathrm{~mm} \mathrm{Hg}$; (g) severe hypoxaemia $\left(\mathrm{PaO}_{2}<50\right.$ $\mathrm{mm} \mathrm{Hg}$ on room air); and (h) a secure diagnosis based on clinical and high-resolution CT (HRCT) data [18].

HRCT images were made within 1 month prior to bronchoscopy. CT scans were analysed by 3 dedicated radiologists (A.C., S.P., and N.S.); divergent opinions were discussed and a shared report was provided.

Each case was discussed during the weekly meetings in a multidisciplinary scenario with the participation of pulmonologists, radiologists, and pathologists. After discussion, a definite diagnosis was suggested; if an inability to achieve a diagnosis was recognized, new investigation or follow-up was proposed.

The patients were deeply sedated with intravenous propofol and remifentanil and intubated with a rigid tracheoscope (Karl Storz GmbH, Tuttlingen, Germany); oxygen was administered continuously, and spontaneous breathing was maintained during the procedure. A flexible cryoprobe measuring $90 \mathrm{~cm}$ in length and $2.4 \mathrm{~mm}$ in diameter was used (Erbokryo CA; Erbe Elektromedizin $\mathrm{GmbH}$, Tübingen, Germany) and the probe was cooled with carbon dioxide $\left(\mathrm{CO}_{2}\right)$, which allowed the temperature in the probe's tip to decrease to $-75^{\circ} \mathrm{C}$ within several seconds. Biopsies were obtained under fluoroscopic guidance using a flexible bronchoscope inserted through the rigid tube into the selected bronchus. Particular attention was devoted to the position of the biopsy; thus, biopsies taken from the same segment were taken in the same position (the same subsegment). Once brought into position, the probe was cooled for approximately $5 \mathrm{~s}$ and then retracted, with the frozen lung tissue attached to the tip of the probe [19]. Each biopsy was performed with a non-inflated Fogarty balloon placed in the lobar bronchus; the balloon was always inflated after biopsy, then immediately deflated in the absence of haemorrhage. Each biopsy sample was collected and processed individually.

Pathologic interpretations, made separately by experts in thoracic pathology (A.D., V.P., M.C., and A.C.), were based on the same features/criteria as used for SLB [20]; analysis of the samples was performed sequentially (from the first to the last sample), and the pathologists reformulated their reports with the addition of each sample. The samples were classified as providing a diagnostic pattern or not ("non-diagnostic pattern"), and divergent opinions were resolved by consensus. The pathologic diagnosis of usual interstitial pneumonia (UIP) was made according to the criteria listed in current guidelines; for the identification of a UIP pattern, the morphologic descriptors were patchy fibrosis, fibroblastic foci, and honeycombing [10,20-23]. Cases of UIP showing a combination of patchy fibrosis and fibroblastic foci, with or without honeycombing, were assigned a rating of "high confidence" in the diagnosis (Fig. 1a); cases of "UIP with low confidence" showed the following: patchy fibrosis plus honeycombing (without fibroblastic foci); fibroblastic foci plus honeycombing (without patchy fibrosis); or just 1 of the 3 main features of UIP (patchy fibrosis or fibroblastic foci or honeycombing) (Fig. 1b). All cases that exhibited pathologic findings suggestive of, or definitive for, an alternative diagnosis were considered "non-UIP" and classified according to the characteristic pathologic features, i.e., non-specific interstitial pneumonia (NSIP), organizing pneumonia, hypersensitivity pneumonitis, sarcoidosis, or other. 

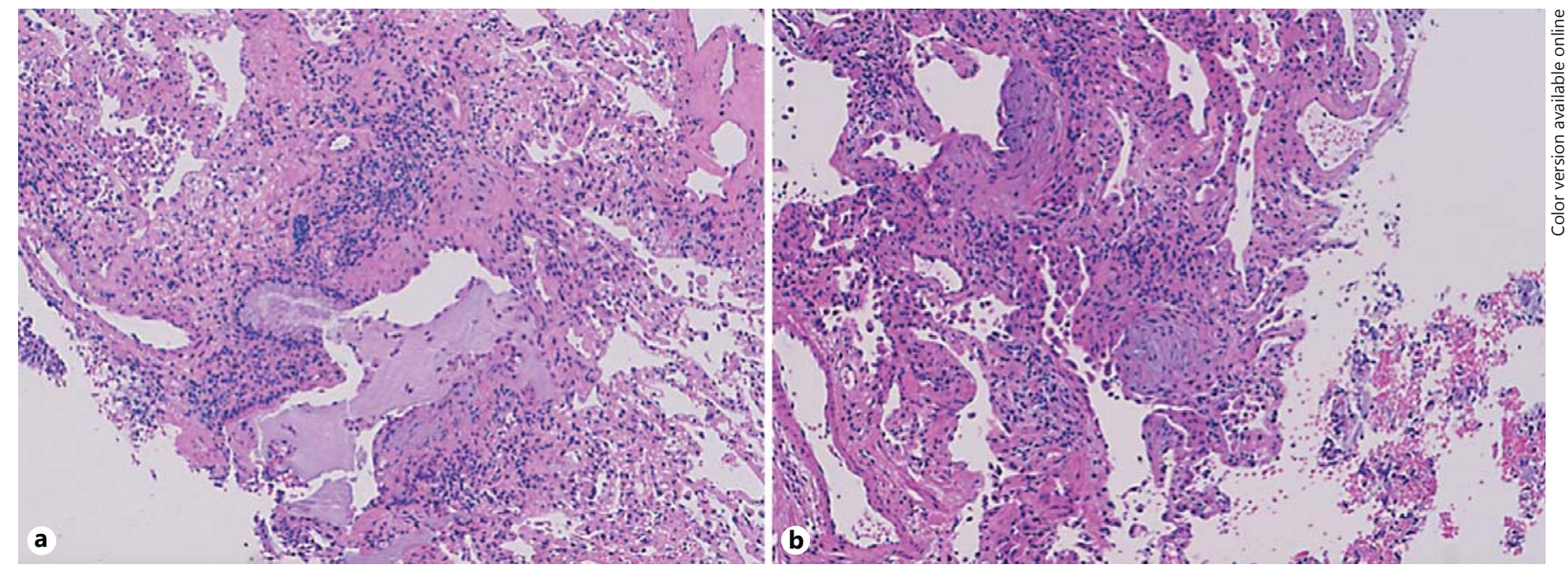

Fig. 1. Different examples of cryobiopsy showing a usual interstitial pneumonia pattern. a Dense scarring obliterating the alveolar architecture and abruptly alternating with relatively normal areas of the lung (patchy fibrosis), some fibroblastic foci, and areas of honeycombing. b Dense fibrosis and fibroblastic foci.

Table 1. Clinical and radiological features of patients submitted to TLCB

\begin{tabular}{lccc}
\hline & $\begin{array}{l}\text { Group A } \\
\text { (only 1 segment) }\end{array}$ & $\begin{array}{l}\text { Group B } \\
\text { (2 different segments) }\end{array}$ & $p$ \\
\hline Cases, $n$ & 22 & 23 & 0.672 \\
Male, $n$ (\%) & $13(59)$ & $15(65)$ & 0.706 \\
Median age (range), years & $65(79-29)$ & $59(74-22)$ & 0.422 \\
Median FVC (range), $\%$ & $85.00(137-49)$ & $90.00(122-55)$ & 0.361 \\
Median DLCO (range), $\%$ & $69.00(121-37)$ & $63.00(100-38)$ & 0.641 \\
Median BMI (range) & $27.50(32.8-19.8)$ & $25.95(33.60-19.00)$ & 0.873 \\
Possible UIP on HRCT, $n(\%)$ & $10(45)$ & $11(48)$ & $12(52)$ \\
Inconsistent UIP on HRCT, $n(\%)$ & $12(55)$ & & \\
\hline
\end{tabular}

TLCB, transbronchial lung cryobiopsy; UIP, usual interstitial pneumonia; HRCT, high-resolution CT.

Statistical Analysis

Statistical analyses were carried out using SPSS Statistics. Data are reported as median and range for continuous variables, and as number and percentage for discrete variables. The different approaches to sampling (strategies $\mathrm{A}$ and $\mathrm{B}$ ) were compared using standard statistical approaches (the $\mathrm{McNemar} \chi^{2}$ or Fisher exact text for categorical variables and the Mann-Whitney $U$ test for continuous variables), and a $p$ value of $<0.05$ was defined as representing a statistically significant difference.

\section{Results}

Twenty-two patients underwent biopsies from the same segment (strategy A) and 23 patients had 2 samples biopsied from one segment and 2 samples from a differ- ent segment of the same lobe (strategy B). The clinical and radiological features of the patients are reported in Table 1 . The time between introduction and removal of the rigid tracheoscope was $20.5 \mathrm{~min}$ (range 11-35) and 18 min (range 12-27) with the 2 different sampling approaches, and no statistically significant differences were found. Also the time between introduction of the probe into the operating channel of the flexible bronchoscope for the first biopsy and its removal after the last biopsy was not statistically different between the 2 groups: 9 min (range 5-21) and 8.5 min (range 5-15) for group A and group B, respectively. All specimens except $12(12 / 151=$ $8 \%$ ) were considered adequate, as they contained alveolated tissue. In 8 patients (18\%), fewer than 4 samples were obtained because the resistance during retrieval of 
Table 2. Diagnostic yield according to the number of samples

\begin{tabular}{lrr}
\hline & Group A $(N=22$ patients $)$ & Group B (N=23 patients) \\
\hline Performing only 1 sample & $23 \%$ not diagnostic $(5 / 22)$ & $39 \%$ not diagnostic $(9 / 23)$ \\
Combining 2 samples (from the same segment) & $9 \%$ not diagnostic $(2 / 22)$ & $35 \%$ not diagnostic $(8 / 23)$ \\
Combining 2 samples (from different segments) & & $4 \%$ not diagnostic (1/23) \\
\hline
\end{tabular}

Table 3. Different pathologic patterns according to the different sampling methods used

\begin{tabular}{llll}
\hline Histologic pattern & $\begin{array}{l}\text { Group A } \\
\text { (same } \\
\text { segment), } n\end{array}$ & $\begin{array}{l}\text { Group B } \\
\text { (different } \\
\text { segments), } n\end{array}$ & $\begin{array}{l}\text { Total } \\
\text { cases }\end{array}$ \\
\hline UIP & 4 & 11 & 15 \\
NSIP & 6 & 1 & 6 \\
OP/NSIP & 1 & 2 & 2 \\
OP & 1 & 3 & 3 \\
Sarcoid & 3 & & 6 \\
Alveolar proteinosis & 1 & & 1 \\
Nodular lymphoid & & & 3 \\
$\quad$ hyperplasia & 3 & & 1 \\
Adenocarcinoma & 1 & & 1 \\
Necrotizing granuloma & 1 & 1 & 1 \\
DAD - fibrotic & 1 & 1 & 1 \\
Langerhans histiocytosis & & 1 & 1 \\
SRIF & & 1 & 1 \\
Bronchiolitis & 1 & 1 \\
Respiratory bronchiolitis & & 1 & 1 \\
GLILD & & & \\
Eosinophilic pneumonia & &
\end{tabular}

UIP, usual interstitial pneumonia; NSIP, non-specific interstitial pneumonia; $\mathrm{OP}$, organizing pneumonia; DAD, diffuse alveolar damage; SRIF, smoking-related interstitial fibrosis; GLILD, granulomatous and lymphocytic interstitial lung disease.

the probe was considered to be excessive (and potentially dangerous). This was also the reason for the single screening failure documented and observed in group $\mathrm{A}$.

The mean size of the samples was $29-30 \mathrm{~mm}^{2}$, while the aggregate biopsy specimen size in each single patient varied from 43 to $286 \mathrm{~mm}^{2}$ (mean $95 \mathrm{~mm}^{2}$ in group A and $115 \mathrm{~mm}^{2}$ in group B). The average size of the first biopsy was 34.58 and $37.81 \mathrm{~mm}^{2}$ in group A and group B, respectively, whereas the average size of the second biopsy was $25.69 \mathrm{~mm}^{2}$ (if performed in the same segment) and 28.07 $\mathrm{mm}^{2}$ (if performed in a different segment); there was no statistically significant difference in size regardless of whether the biopsy was performed in the same segment or in different segments.
Table 4. Relationship between histopathologic descriptors of the UIP pattern and specific diagnostic samples

\begin{tabular}{lllll}
\hline & \multicolumn{3}{l}{ Sample Sample Sample Sample } \\
& I & II & III & IV \\
& & & & \\
\hline $\begin{array}{l}\text { Group A } \\
\text { UIP }\end{array}$ & & & & \\
$\quad$ PF + FF + HC & 1 & & $\mathbf{1}$ & \\
$\quad$ PF + FF & 2 & & 1 & \\
\hline $\begin{array}{l}\text { Group B } \\
\text { UIP }\end{array}$ & & & & \\
$\quad$ PF + FF + HC & 5 & $\mathbf{1}$ & $\mathbf{4}$ & $\mathbf{1}$ \\
$\quad$ PF + FF + HC + ancillary & & 1 & 1 & \\
$\quad$ findings of HP & 0 & & & \\
$\quad$ PF + FF & 2 & & 2 & 1
\end{tabular}

UIP, usual interstitial pneumonia; PF, patchy fibrosis; FF, fibroblastic foci; HC, honeycombing; HP, hypersensitivity pneumonia.

In group $\mathrm{A}$, the patients underwent 4 biopsies from the same segment (A1, A2, A3, A4); in group B, the patients underwent 4 biopsies, $\mathrm{B} 1$ and $\mathrm{B} 2$ from one segment and B3 and B4 from a different segment. The mean diagnostic yield of cryobiopsy taking only 1 sample (therefore combining A1 and B1) was $69 \%$ (mean rate of non-diagnostic appearances: $31 \%$ ). Cases in which a pathologic pattern could be identified with only 1 sample were characterized by homogeneous and typical morphologic alterations (NSIP, organizing pneumonia, sarcoidosis, or adenocarcinoma). When a second biopsy was performed in the same segment as well (therefore combining samples A1 + $\mathrm{A} 2$ and samples B1 + B2), the mean diagnostic yield increased to $78 \%$, but with no statistically significant difference ( $p=0.340)$; however, if the second biopsy was taken from a separate segment (therefore combining samples B1 + B3), the diagnostic yield increased to $96 \%(p=0.004$, McNemar $\chi^{2}$ test) (Table 2). Furthermore, when the relationship between biopsy size and diagnostic yield was evaluated using simple logistic regression, there was no 


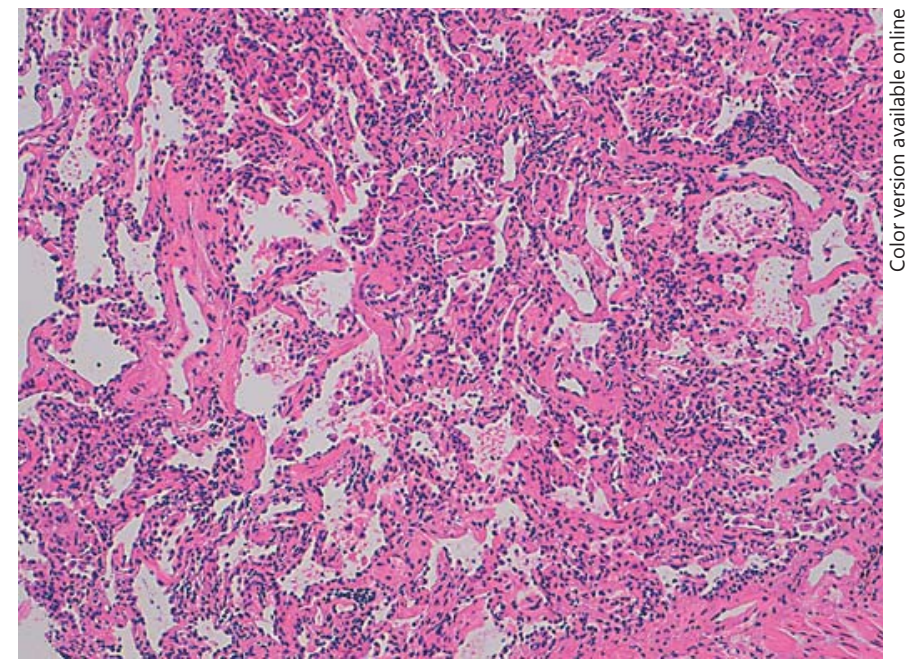

Fig. 2. Non-specific interstitial pneumonia pattern. A temporally homogeneous alveolar septal fibrosis is evident.

statistically significant relationship regardless of whether biopsies were performed in the same segment $(p=0.319)$ or in different segments $(p=0.279)$.

The different pathologic patterns observed are described in Table 3. The UIP pattern was identified in 15 cases, and was more frequent in group B (different segments); all 3 morphologic descriptors of UIP were identified in 8 cases, whereas in 7 cases we identified only 2 histopathologic descriptors of UIP: patchy fibrosis and fibroblastic foci [24] (Table 4). For 2 cases identified as "chronic hypersensitivity pneumonitis with UIP pattern," the diagnosis was obtained analysing samples obtained from different segments by the presence of ancillary features (foci of intra-alveolar organizing pneumonia, loosely formed microgranulomas, and lymphocytic bronchiolitis). The NSIP pattern was detected in 8 patients (Fig. 2). Each case was discussed, and a final diagnosis was reached; in group B, no surgical biopsy was deemed necessary after multidisciplinary discussion, whereas in group A, a surgical biopsy was performed in 2 cases.

Regarding safety, no severe bleeding was observed, only mild-to-moderate bleeding ( 2 cases in group A and 2 cases in group B). Bleeding was defined as "mild" (grade 1) if it required suction to clear but no other endoscopic procedures, as "moderate" (grade 2) if it required endoscopic procedures such as bronchial occlusion-collapse and/or instillation of ice-cold saline, and as "severe" (grade 3) if "causing haemodynamic or respiratory instability, requiring tamponade or other surgical interven-

Transbronchial Lung Cryobiopsy in

Diffuse Parenchymal Lung Disease tions, transfusions, or admission to intensive care unit" [25]. Pneumothorax was documented in $16 \%$ of the cases ( 1 case in group A and 6 cases in group B) $(p=0.096)$; fragments of pleura were found only in patients from whom 4 specimens were obtained ( 7 in group A and 6 in group B [13/45 =29\%]). No death, acute lung injury, persistent fever, prolonged air leak, pneumonia/empyema, or other adverse events were observed after TLCB in the 2 groups [26]. When the relationship between biopsy size and development of complications was evaluated (pneumothorax together with bleeding) by simple logistic regression, there was no statistically significant relationship found regardless of whether biopsies were performed in the same segment $(p=0.973)$ or in different segments $(p=0.147)$.

\section{Discussion}

TLCB has an increasing diagnostic value for patients with DPLD [5-16]. Multiple biopsies are undertaken in an effort to reduce sampling error, but there are no published studies considering the implications of such data; the relationship between the number of samples retrieved and diagnostic yield is not known, and, in particular, it is uncertain whether sampling from 2 segments is more sensitive than sampling from only 1 segment. Data on the different strategies adopted to sample lung tissue are still missing in the literature; the majority of studies reported retrieving lung tissue from 1 segment, and only a minority collected lung samples from different segments of the same lobe. No prospective studies have been published yet comparing these 2 approaches in terms of diagnostic yield and rates of complications, and no data are yet available on the diagnostic value of TLCB from 2 lobes, although there are concerns about an increase in complications (mainly pneumothorax). In this first study, we prospectively tested the diagnostic yield of 2 different methods of obtaining TLCB samples from the same lobe. A multicentre study on diagnostic yield and complications of TLCB is ongoing, and its results may help to standardize this technique even more in the future.

This study shows that taking 2 samples instead of only 1 sample can increase the diagnostic yield of cryobiopsy in DPLD; however, this increase appears statistically significant only when the 2 samples are taken from different segments. The number of NSIP patterns was higher in group $A$ than in group $B$, whereas the UIP pattern was recognized at a higher rate in group $B(p<0.05)$. This could be due to the unequal distribution of clinical cases 
related to the small number of patients recruited. However, among those with an NSIP pattern, 1 case had a definite diagnosis of idiopathic pulmonary fibrosis (IPF; after a UIP pattern had been identified in SLB samples and following multidisciplinary discussion), 1 case was diagnosed as chronic hypersensitivity pneumonitis (after having considered the meaning of lymphocytosis in bronchoalveolar lavage and a more precise interview), and 1 case was classified as interstitial pneumonia with autoimmune features after multidisciplinary discussion of laboratory, clinical, and morphologic features. These observations indicate that when a pattern of fibrosing NSIP is documented on cryobiopsy samples obtained from the same lobar segment, sampling error is a major consideration, with - at least in a minority of cases - a final diagnosis of IPF made after multidisciplinary discussion. This consideration provides a strong conceptual basis for the practice of performing multiple biopsies in different segments.

Regarding sample size, previous studies have reported cryobiopsy samples varying in size between 9 and $64.2 \mathrm{~mm}^{2}$ in surface area and aggregate biopsy specimen sizes varying between 15 and $130 \mathrm{~mm}^{2}$, which is far larger than the sizes of tissue samples retrieved using conventional forceps $[6-15,17]$. The cryoprobes used are of different sizes ( 1.9 or $2.4 \mathrm{~mm}$ ) and the freezing time (2-8 s) is also different between reports in the current literature. The longer freezing times and larger diameters of the cryoprobes correlate positively with the weight and diameter of cryobiopsy samples. Concerning the dependency on freezing time, a significant increase in biopsy weight was observed after $3 \mathrm{~s}$ with the $1.9-\mathrm{mm}$ cryoprobe compared with a freezing time of $2 \mathrm{~s}$, and after 2 $\mathrm{s}$ with the $2.4-\mathrm{mm}$ probe compared with a freezing time of $1 \mathrm{~s}$. In the studies published so far, sample sizes appear to be bigger when larger cryoprobes or freezing times $>4$ $\mathrm{s}$ are used. A freezing time of 3-6 s was considered adequate for larger probes $(2.4 \mathrm{~mm})$, while a longer freezing time up to $7-8 \mathrm{~s}$ was considered adequate for smaller probes [27].

In our series, the 2.4-mm cryoprobe was used and was cooled for approximately $5 \mathrm{~s}$. Furthermore, the specimens obtained by cryoprobe often included structures from the periphery of the secondary pulmonary lobule; in fact, pleura was detected in 13 out of 45 cases (29\%). Conventional transbronchial lung biopsy provides specimens with these peripheral structures in less than $10 \%$ of cases; TLCB is able to obtain larger samples of peripherally located lung tissue, thereby significantly increasing its diagnostic yield and specificity in IPF or chronic hy- persensitivity pneumonia, and may resolve the difficulties met using conventional transbronchial lung biopsy [28]. This assertion, already hypothesized in our previous study [10], is confirmed by the observation that the 3 morphologic descriptors of UIP (patchy fibrosis, fibroblastic foci, and honeycombing) were identified in 8 cases (53\%), while only 2 descriptors, patchy fibrosis and fibroblastic foci [24], were identified in 7 cases (47\%). The increased diagnostic yield of TLCB compared with transbronchial biopsy appears to be robust, irrespective of the volume of tissue acquired by TLCB. We evaluated the relationship between sample size and diagnostic yield, and we did not observe any statistically significant relationship regardless of whether sampling was performed in the same segment or in different segments. Our study demonstrated a mean diagnostic yield of cryobiopsy of $69 \%$ (mean rate of non-diagnostic appearances: $31 \%$ ) when taking only 1 sample; when a second biopsy was performed as well, the mean diagnostic yield increased to $78 \%$ (if the biopsy was performed in the same segment) or $96 \%$ (if the biopsy was performed in a separate segment). This diagnostic yield of cryobiopsies is generally greater than what has previously been reported, varying between studies from $70-95 \%$ with a $2.4-\mathrm{mm}$ probe to $79-80 \%$ with a $1.9-\mathrm{mm}$ probe [27]. The overall diagnostic yield derived from the 15 studies investigated in our previous meta-analysis [29], in which biopsies were taken mostly from within the same segment, was 0.81 (0.75-0.87), which is well below the $96 \%$ observed in the present study.

Transbronchial lung biopsy via cryoprobe was completed in about $20 \mathrm{~min}$, compared to a reported time of 45 min for surgical biopsy [30]. In some patients, excessive resistance during retrieval of the probe can be observed. One hypothesis that could explain this phenomenon is that the cryoprobe is lodged in small bronchi (with cartilage plates in the wall) and not more distally, mainly due to the presence of bronchomalacia, and therefore the tissue (consisting mainly of the bronchial wall) captured by the freezing process may not be extracted without a strong tug. In agreement with this hypothesis is the fact that fragments of pleura were identified only in cases in which all 4 samples were taken. In this study, the use of smaller cryoprobes was not allowed, but this inconvenience could be avoided (as assessed in our daily practice) using such probes $(1.9 \mathrm{~mm})$.

It has recently been demonstrated that TLCB is a safe procedure, with lower complication and mortality rates compared to SLB; patient groups with DPLD undergoing TLCB have a significantly lower mortality rate following 
the procedure compared to those undergoing videoassisted thoracoscopic surgery, and the overall mortality rate with this procedure among approximately 1,000 patients is negligible (0.1\%) [29]. In this study, no deaths or acute lung injuries were observed. No severe bleeding occurred, and none of these patients needed an additional intervention, mostly due to the preventive use of a Fogarty balloon, which was prophylactically placed in the lobar bronchus near the biopsy segment and routinely inflated after sampling to minimize the consequence of haemorrhage. The routine preventive use of Fogarty balloons was first described by our group in 2008, and it seems to be a very efficient way to control bleeding when large endoscopic lung biopsies are made [31, 32]. Moreover, it is likely that the haemostatic effects of cooling contribute to the low incidence of significant bleeding [29]. Pneumothorax occurred in $16 \%$ of the patients, with no statistically significant difference when performing biopsies in different segments. We did not observe statistically significant relationships between complications and sample size, whether sampling in the same segment or in different segments.

The study has some limitations. The number of patients enrolled was small and the identification of statistically significant group differences was difficult. Indeed, the significant improvement in diagnostic yield with 2 -segment sampling was demonstrable only because, by chance, the initial biopsy was more frequently non-diagnostic in group B; the improvement was nonetheless statistically significant. The absence of SLB in the vast majority of cases limited our ability to compare the accuracy of TLCB in terms of its sensitivity and specificity for the diagnosis of fibrotic DPLD. A further issue, common to current cryobiopsy studies, was the absence of a diagnos- tic reference standard (a histological diagnosis by SLB). However, a "definitive" protocol, in which cryobiopsy is followed by a diagnostic surgical biopsy, raises serious ethical concerns. In many cases, cryobiopsy data allow the formulation of a definitive diagnosis, and the subsequent performance of a surgical biopsy merely results in avoidable morbidity and mortality, without any added diagnostic value.

In conclusion, TLCB has a good diagnostic yield in fibrotic interstitial pneumonia. Pathologists are generally confident in their ability to make diagnoses using essentially the same criteria as for SLB. In order to have a higher diagnostic yield in this context, 1 sample from one segment and 1 sample from a different adjacent segment seems to be the best strategy to follow. TLCB with a diagnosis of fibrotic DPLD appears safe and feasible; pneumothorax is the main complication, and the preventive use of a Fogarty catheter might be recommended in all cases to prevent bleeding. The morphologic descriptors of complex histopathologic patterns may be identified in samples that are smaller than those obtained by SLB but significantly bigger than those collected by conventional transbronchial lung biopsy. Finally, in this study, at least 21 patients $(47 \%)$ had radiological features (possible UIP pattern on HRCT) requiring, according to the guidelines, an SLB [24-26, 28-33]. TLCB allowed avoiding this invasive procedure, which causes significant morbidity and mortality [34-36], in almost all of these cases [37-39].

\section{Financial Disclosure and Conflicts of Interest}

None of the authors have any potential conflicts of interest with any companies/organizations whose products or services may be discussed in this paper.

\section{References}

1 Poletti V, Casoni GL, Gurioli C, Ryu JH, Tomassetti S: Lung cryobiopsies: a paradigm shift in diagnostic bronchoscopy? Respirology 2014;19:645-646.

2 Hernández-González F, Lucena CM, Ramírez J, Sánchez M, Jimenez MJ, Xaubet A, Sellares J, Agustí C: Cryobiopsy in the diagnosis of diffuse interstitial lung disease: yield and costeffectiveness analysis (in English, Spanish). Arch Bronconeumol 2015;51:261-267.

3 Poletti V, Benzaquen S: Transbronchial cryobiopsy in diffuse parenchymal lung disease. A new star in the horizon. Sarcoidosis Vasc Diffuse Lung Dis 2014;31:178-181.
4 Mikolasch TA, Porter JC: Transbronchial cryobiopsy in the diagnosis of interstitial lung disease: a cool new approach. Respirology 2014;19:623-624.

5 Maldonado F, Moua T, Skalski J: Parenchymal cryobiopsies for interstitial lung diseases: a step forward in disease management. Respirology 2014;19:773-774.

6 Poletti V, Gurioli C, Piciucchi S, Rossi A, Ravaglia C, Dubini A, Asioli S, Casoni GL: Intravascular large $B$ cell lymphoma presenting in the lung: the diagnostic value of transbronchial cryobiopsy. Sarcoidosis Vasc Diffuse Lung Dis 2015;31:354-358.
7 Griff S, Schönfeld N, Ammenwerth W, Blum TG, Grah C, Bauer TT, Grüning W, Mairinger T, Wurps H: Diagnostic yield of transbronchial cryobiopsy in non-neoplastic lung disease: a retrospective case series. BMC Pulm Med 2014;14:171.

8 Pourabdollah M, Shamaei M, Karimi S, Karimi M, Kiani A, Jabbari HR: Transbronchial lung biopsy: the pathologist's point of view. Clin Respir J 2016;10:211-216.
Transbronchial Lung Cryobiopsy in Diffuse Parenchymal Lung Disease 
9 Pajares V, Puzo C, Castillo D, Lerma E, Montero MA, Ramos-Barbón D, Amor-Carro O, Gil de Bernabé A, Franquet T, Plaza V, Hetzel J, Sanchis J, Torrego A: Diagnostic yield of transbronchial cryobiopsy in interstitial lung disease: a randomized trial. Respirology 2014; 19:900-906.

10 Casoni GL, Tomassetti S, Cavazza A, Colby TV, Dubini A, Ryu JH, Carretta E, Tantalocco P, Piciucchi S, Ravaglia C, Gurioli C, Romagnoli M, Gurioli C, Chilosi M, Poletti V: Transbronchial lung cryobiopsy in the diagnosis of fibrotic interstitial lung diseases. PLoS One 2014;9:e86716.

11 Dincer HE, Amin K, Gibson H: A new tool in diagnosis of lung diseases: cryoprobe lung biopsy: case series and the review of literature. Clin Pulm Med 2014;21:181-184.

12 Yarmus L, Akulian J, Gilbert C, Illei P, Shah P, Merlo C, Orens J, Feller-Kopman D: Cryoprobe transbronchial lung biopsy in patients after lung transplantation: a pilot safety study. Chest 2013;143:621-626.

13 Kropski JA, Pritchett JM, Mason WR, Sivarajan L, Gleaves LA, Johnson JE, Lancaster LH, Lawson WE, Blackwell TS, Steele MP, Loyd JE, Rickman OB: Bronchoscopic cryobiopsy for the diagnosis of diffuse parenchymal lung disease. PLoS One 2013;8:e78674.

14 Fruchter O, Fridel L, Rosengarten D, Rahman NA, Kramer MR: Transbronchial cryobiopsy in immunocompromised patients with pulmonary infiltrates: a pilot study. Lung 2013; 191:619-624.

15 Pajares V, Torrego A, Puzo C, Lerma E, Gil de Bernabé MA, Franquet T: Transbronchial lung biopsy using cryoprobes (in Spanish). Arch Bronconeumol 2010;46:111-115.

16 Griff S, Ammenwerth W, Schönfeld N, Bauer TT, Mairinger T, Blum TG, Kollmeier J, Grüning W: Morphometrical analysis of transbronchial cryobiopsies. Diagn Pathol 2011;6:53.

17 Babiak A, Hetzel J, Krishna G, Fritz P, Moeller P, Balli T, Hetzel M: Transbronchial cryobiopsy: a new tool for lung biopsies. Respiration 2009;78:203-208.

18 Sverzellati N, Lynch DA, Hansell DM, Johkoh T, King TE Jr, Travis WD: American Thoracic Society-European Respiratory Society Classification of the Idiopathic Interstitial Pneumonias: advances in knowledge since 2002. Radiographics 2015;35:1849-1871.

19 Tomic R, Podgaetz E, Andrade RS, Dincer HE: Cryotechnology in diagnosing and treating lung diseases. J Bronchology Interv Pulmonol 2015;22:76-84.
20 Larsen BT, Colby TV: Update for pathologists on idiopathic interstitial pneumonias. Arch Pathol Lab Med 2012;136:1234-1241.

21 Chilosi M, Zamò A, Doglioni C, Reghellin D, Lestani M, Montagna L, Pedron S, Ennas MG, Cancellieri A, Murer B, Poletti V: Migratory marker expression in fibroblast foci of idiopathic pulmonary fibrosis. Respir Res 2006; 7:95.

22 Chilosi M, Poletti V, Murer B, Lestani M, Cancellieri A, Montagna L, Piccoli P, Cangi G, Semenzato G, Doglioni C: Abnormal re-epithelialization and lung remodeling in idiopathic pulmonary fibrosis: the role of $\Delta \mathrm{N}$ p63. Lab Invest 2002;82:1335-1345.

23 Colby TV: Pulmonary pathology: LC22-1 diagnosis of idiopathic pulmonary fibrosis (IPF): histologic and HRCT diagnosis. Pathology 2014;46(suppl 2):S36.

24 Raghu G, Collard HR, Egan JJ, et al; ATS/ERS/ JRS/ALAT Committee on Idiopathic Pulmonary Fibrosis: An official ATS/ERS/JRS/ ALAT statement: idiopathic pulmonary fibrosis: evidence-based guidelines for diagnosis and management. Am J Respir Crit Care Med 2011;183:788-824.

25 Ernst A, Eberhardt R, Wahidi M, Becker HD Herth FJ: Effect of routine clopidogrel use on bleeding complications after transbronchial biopsy in humans. Chest 2006;129:734-737.

26 Singhal S, Ferraris VA, Bridges CR, Clough ER, Mitchell JD, Fernando HC, Shrager JB: Management of alveolar air leaks after pulmonary resection. Ann Thorac Surg 2010;89: 1327-1335.

27 Poletti V, Hetzel J: Transbronchial cryobiopsy in diffuse parenchymal lung disease: need for procedural standardization. Respiration 2015;90:275-278.

28 Tomassetti S, Cavazza A, Colby TV, Ryu JH, Nanni O, Scarpi E, Tantalocco P, Buccioli M, Dubini A, Piciucchi S, Ravaglia C, Gurioli C, Casoni GL, Gurioli C, Romagnoli M, Poletti $\mathrm{V}$ : Transbronchial biopsy is useful in predicting UIP pattern. Respir Res 2012;13:96.

29 Ravaglia C, Bonifazi M, Wells AU, Tomassetti S, Gurioli C, Piciucchi S, Dubini A, Tantalocco P, Sanna S, Negri E, Tramacere I, Ventura VA, Cavazza A, Rossi A, Chilosi M, La Vecchia C, Gasparini S, Poletti V: Safety and diagnostic yield of transbronchial lung cryobiopsy in diffuse parenchymal lung diseases: a comparative study versus video-assisted thoracoscopic lung biopsy and a systematic review of the literature. Respiration 2016;91: 215-227.
30 Ayed AK, Raghunathan R: Thoracoscopy versus open lung biopsy in the diagnosis of interstitial lung disease: a randomised controlled trial. J R Coll Surg Edinb 2000;45:159-163.

31 Hagmeyer L, Theegarten D, Wohlschläger J, Treml M, Matthes S, Priegnitz C, Randerath WJ: The role of transbronchial cryobiopsy and surgical lung biopsy in the diagnostic algorithm of interstitial lung disease. Clin Respir J 2016;10:589-595.

32 Casoni GL, Gurioli C, Chhajed PN, Chilosi M, Zompatori M, Olivieri D, Poletti V: The value of transbronchial lung biopsy using jumbo forceps via rigid bronchoscope in diffuse lung disease. Monaldi Arch Chest Dis 2008;69: 59-64.

33 Monaghan H, Wells AU, Colby TV, du Bois RM, Hansell DM, Nicholson AG: Prognostic implications of histologic patterns in multiple surgical lung biopsies from patients with idiopathic interstitial pneumonias. Chest 2004; 125:522-526.

34 Medford AR: Linear endobronchial ultrasound learning curve: hard to predict. Chest 2014; 146:e221.

35 Travis WD, Costabel U, Hansell DM, et al; ATS/ERS Committee on Idiopathic Interstitial Pneumonias: An official American Thoracic Society/European Respiratory Society statement: update of the international multidisciplinary classification of the idiopathic interstitial pneumonias. Am J Respir Crit Care Med 2013;188:733-748.

36 Han Q, Luo Q, Xie JX, Wu LL, Liao LY, Zhang $\mathrm{XX}$, Chen RC: Diagnostic yield and postoperative mortality associated with surgical lung biopsy for evaluation of interstitial lung diseases: a systematic review and meta-analysis. J Thorac Cardiovasc Surg 2015;149:13941401.

37 Tomassetti S, Wells AU, Costabel U, Cavazza A, Colby TV, Rossi G, Sverzellati N, Carloni A, Carretta E, Buccioli M, Tantalocco P, Ravaglia C, Gurioli C, Dubini A, Piciucchi S, Ryu $\mathrm{JH}$, Poletti V: Bronchoscopic lung cryobiopsy increases diagnostic confidence in the multidisciplinary diagnosis of idiopathic pulmonary fibrosis. Am J Respir Crit Care Med 2016;193:745-752.

38 Poletti V, Ravaglia C, Tomassetti S: Transbronchial cryobiopsy in diffuse parenchymal lung diseases. Curr Opin Pulm Med 2016;22: 289-296.

39 Colby TV, Tomassetti S, Cavazza A, Dubini A, Poletti V: Transbronchial cryobiopsy in diffuse lung disease: update for the pathologist. Arch Pathol Lab Med 2016, Epub ahead of print. 